\title{
COMORBILIDAD ASOCIADA AL SÍNDROME DE VEJIGA HIPERACTIVA
}

\author{
David Castro Díaz', Pablo Rebollo² y Diego González-Segura Alsina³.
}

'Servicio de Urología. Hospital Universitario de Canarias. 2BAP Health Outcomes Research, S.L. Oviedo.

${ }^{3}$ Departamento Médico. Almirall S.A. Barcelona. España.

Resumen.- OBJETIVOS: Conocer el porcentaje de pacientes con vejiga hiperactiva (VH) que acuden a consultas de urología o ginecología y que presentan patologías asociadas que complican su condición clínica.

MÉTODOS: Estudio epidemiológico, de corte transversal, multicéntrico, en práctica clínica habitual en el que se incluyeron a 1.659 pacientes con $\mathrm{VH}$, en consultas de urología o ginecología. Se recogieron datos demográficos, antropométricos, presencia de trastornos crónicos (hipertensión, diabetes, accidentes cerebro-vasculares, cardiopatía isquémica, enfermedades respiratorias, deterioro cognitivo, catarata o glaucoma, disminución de la movilidad y estreñimientol, presencia de patologías asociadas (caídas, infecciones del tracto urinario inferior, infecciones de la piel en el área genital, depre-

\section{CORRESPONDENCIA}

David Castro Díaz

Servicio de Urología

Hospital Universitario de Canarias

C/Ofra s/n, La Laguna

38320 Santa Cruz de Tenerife. (España).

dcastro@teide.net

Trabajo recibido: 10 de septiembre 2008. sión, disfunción sexual y trastornos del sueño), presencia de síntomas de incontinencia urinaria y su impacto en la vida según puntuación del ICIQ-UI SF.

RESULTADOS: La edad media (D.E.) de la muestra estudiada fue 59,2 (12,9) años y el 73,6\% eran mujeres. La mayoría vivían en el ámbito urbano $(67,3 \%)$, tenían estudios primarios o no tenían estudios $(56,8 \%)$ y tenían trabajo activo o eran amas de casa $(66,2 \%)$. Las patologías asociadas a la VH más frecuentes fueron las infecciones en la piel del área genital, las infecciones del tracto urinario inferior y los trastornos del sueño. En los varones es más frecuente la disfunción sexual; y en las mujeres, las infecciones urinarias y de la piel del área genital y la depresión. El impacto en el paciente de estos trastornos, se evidencia por la mayor puntuación en el cuestionario ICIQ-UI SF de los pacientes que los padecen, respecto a los que no.

CONCLUSIONES: La presencia de patologías asociadas en pacientes con VH, es frecuente tanto en varones como en mujeres y se asocia a mayor impacto en la vida del paciente. Parece relevante prestar atención a su detección en la historia clínica del paciente que consulta por síntomas de $\mathrm{VH}$ y/o incontinencia urinaria.

Palabras clave: Vejiga hiperactiva. Comorbilidad. Calidad de vida.

Summary.- OBJECTIVES: To estimate the percentage of patients with Overactive Bladder (OB) attending urology or gynaecology outpatient clinics who show associated diseases which complicate their clinical state and quality of life.

METHODS. This is an epidemiologic, cross-sectional and multi-centre real world trial. 1,659 patients with $O B$ were studied in urology or gynaecology outpatient clinics. Demographic data, anthropometric data, presence 
of chronic diseases (hypertension, diabetes, cerebrovascular disease, ischemic cardiopathy, chronic obstructive pulmonary disease, cognitive damaged, cataract, movement disability and constipation), presence of associated diseases (falls, lower urinary tract infections, genital skin infections, depression, sexual dysfunction and sleep disordersl, presence of urinary incontinence symptoms and impact on quality of life according to ICIQ-UI SF score.

RESULTS. Mean age (S.D) of studied sample was 59.2 (12.9) years and $73.6 \%$ were female. Most patients lived in urban settings (67.3\%), had primary studies or less (56.8\%) and had active work or at home work (66.2\%). Most frequent diseases associated to OB were genital skin infections, lower urinary tract infections and sleep disorders. Sexual dysfunction was more frequent in males than in females; lower urinary tract infections, genital skin infections and depression were more frequent in females than in males. Patients with these diseases showed higher ICIQ-UI SF scores than patients without them, indicating higher impact.

CONCLUSIONS. Presence of associated diseases in patients with $O B$ is frequent in males and females and it is associated to higher impact in patients' life. It seems relevant to pay attention in detecting them through the medical history of patients consulting about $O B$ or urinary incontinence symptoms.

Keywords: Overactive bladder. Comorbidity. Quality of life.

\section{INTRODUCCIÓN}

La vejiga hiperactiva $(\mathrm{VH})$ es un trastorno de la función vesical. Los síntomas más característicos son la urgencia miccional, la incontinencia de urgencia, y la frecuencia miccional, consecuencia de la presencia involuntaria de contracciones en la vejiga durante el proceso de llenado (1-2).

La VH es una patología frecuente en la población adulta, y es cada vez más habitual en las consultas de atención primaria, urología y ginecología. Se trata de una patología infradiagnosticada ya que los pacientes son reticentes a acudir a la consulta médica (3). Clásicamente se consideraba que la $\mathrm{VH}$ se podía diagnosticar siempre que el paciente presentara los síntomas de urgencia miccional y/o frecuencia miccional $y / o$ incontinencia de urgencia, sin causa aparente por otra patología o condición metabólica. En el año 2002, la International Continence Society (ICS) modificó la definición considerándose diagnóstico de $\mathrm{VH}$ cuando existe urgencia miccional, con o sin incontinencia de urgencia, pudiendo a menudo asociarse a frecuencia miccional y nicturia (4).
En un estudio epidemiológico transversal con una muestra poblacional de 1669 sujetos de 40 años o más se detectó una elevada prevalencia de $\mathrm{VH}$. En el 21,5\% (IC 95\% 19,5-22,5) de la población estudiada se registró la presencia de urgencia miccional con o sin incontinencia de urgencia. La prevalencia de sintomatología compatible con $\mathrm{VH}$ fue del $25,6 \%$ en mujeres, significativamente superior a la referida entre los hombres $(17,4 \%)$. La prevalencia global fue máxima en los pacientes de mayor edad. Analizado por género, sólo en las mujeres aumentó la prevalencia de $\mathrm{VH}$ de forma progresiva y estadísticamente significativa con la edad. La prevalencia de $\mathrm{VH}$ ajustada a la población española se situó en un 19,9\% (IC 95\% 18,0-21,8), siendo superior entre las mujeres $(23,6 \%)$ que en los hombres $(15,4 \%)(5)$.

La vejiga hiperactiva tiene un gran impacto en la calidad de vida de los pacientes, tanto en hombres como en mujeres afectando a su actividad diaria, al trabajo, a la actividad física, a la función sexual y al sueño (6). Varios estudios han demostrado que los pacientes que padecen $\mathrm{VH}$ con incontinencia urinaria, tienen peor calidad de vida relacionada con la salud que los pacientes diabéticos $(1,7-8)$. Aunque los síntomas de la incontinencia son probablemente los más evidentes y que al parecer afectan más al paciente, el impacto de la frecuencia y urgencia urinarias no deben ser ignorados. En un estudio realizado por Milsom y colaboradores, el $65 \%$ de los hombres y el $67 \%$ de las mujeres con $\mathrm{VH}$ afirmaron que la patología en estudio les afectaba en su vida diaria (9). Estudios recientes con diseño metodológico adecuado confirman que la $\mathrm{VH}$ sin incontinencia también influye en la calidad de vida de forma negativa (10).

La VH asociada a la incontinencia urinaria, además de afectar a la calidad de vida del paciente, puede relacionarse con otras patologías, como infecciones del tracto urinario inferior, infecciones de la piel, trastornos del sueño, depresión y disfunción sexual e incluso aumentar el riesgo de caídas y fracturas recurrentes (11). Un estudio demostró que las mujeres con urgencia urinaria tenían el riesgo de sufrir caídas aumentado en un $26 \%$ y de sufrir fracturas vertebrales en un 34\% (12). Dos estudios demostraron que existe una estrecha relación entre la $\mathrm{VH}$ y la depresión (13-14), aproximadamente el 30\% de todos los pacientes con VH tenían depresión o la habían padecido.

Todas estas patologías complican la condición clínica del paciente y empeoran su calidad de vida, mientras que incrementan el impacto clínico y económico de la $\mathrm{VH}(15)$. 
Por todo lo expuesto anteriormente, y debido al continuo aumento de la $\mathrm{VH}$ y de las patologías asociadas, se propone el presente estudio para conocer el porcentaje de pacientes con vejiga hiperactiva que acuden a consultas de urología o ginecología y que presentan patología asociada (infecciones del tracto urinario inferior, infecciones de la piel, depresión, disfunción sexual, trastornos del sueño y caídas y fracturas).

\section{SUJETOS Y MÉTODO}

Estudio epidemiológico, de corte transversal, multicéntrico, en condiciones de práctica clínica habitual. Se incluyeron 1.659 pacientes que acudieron de forma consecutiva, durante 3 meses, a una de las consultas externas de urología o ginecología participantes en el estudio. Los pacientes incluidos eran mayores de 18 años, de ambos sexos, con síntomas de $\mathrm{VH}$ de acuerdo a la puntuación en el Cuestionario de Autoevaluación del Control de la Vejiga (CACV) (16) y otorgaron su consentimiento informado por escrito. Resultaron excluidas las mujeres embarazadas, con prolapso genital mayor de grado II, y los pacientes con infección urinaria activa $y / o$ con hematuria.

Para cada paciente se recogieron datos demográficos (edad, sexo, ámbito de residencia, nivel de estudios, situación laboral, número de hijos, número de partos vaginales y menopausia); datos antropométricos (peso, talla y perímetro de cintura); presencia de trastornos crónicos (hipertensión, diabetes, accidentes cerebro-vasculares, cardiopatía isquémi$\mathrm{ca}$, enfermedades respiratorias, deterioro cognitivo, catarata o glaucoma, disminución de la movilidad por alguna condición médica y estreñimiento); presencia de patologías asociadas (caídas, infecciones del tracto urinario inferior, infecciones de la piel en el área genital, depresión, disfunción sexual y trastornos del sueño); presencia de síntomas de incontinencia urinaria y su impacto en la vida de la paciente (según la puntuación del cuestionario ICIQ-UI SF).

El cuestionario de autoevaluación del control de la vejiga (CACV) es la versión española del Bladder Health Questionnaire (BHQ), cuestionario diseñado por un grupo europeo de expertos en disfunciones del tracto urinario inferior, con el objetivo de identificar a través de su auto-cumplimentación a las personas con VH. Es un sencillo cuestionario, con tan sólo 8 ítems agrupados en dos escalas ("molestias" $y$ "síntomas") que fue recientemente validado en España (16). La puntuación puede variar entre 0 y 12 puntos, siendo el punto de corte propuesto para detección de VH, 6 ó más puntos. En el presente estudio se empleó su puntuación como criterio de inclusión: sólo los pacientes con síntomas positivos de $\mathrm{VH}$ fueron incluidos en el estudio.

El International Consultation on Incontinence Questionnaire-Short Form (ICIQ-UI SF), es un cuestionario diseñado para evaluar la presencia de síntomas de incontinencia urinaria y su impacto en la vida del paciente. Ha sido formalmente validado en España (17). El ICIQ-UI SF consta de dos grupos de preguntas:

1) 3 preguntas, sobre frecuencia, cantidad y afectación de la calidad de vida, con cuyas respuestas se calcula la puntuación del cuestionario que puede variar entre 0 (continente) y 21 puntos (máximo grado de IU);

2) ocho preguntas que se refieren a las situaciones en las que se produce la IU y que permiten orientar el diagnóstico del tipo de IU (de esfuerzo, de urgencia o mixta).

\section{Análisis estadístico}

Las variables categóricas se describen mediante frecuencias absolutas y relativas, incluyendo el intervalo de confianza del $95 \%$. Para la descripción de las variables continuas se utiliza la media y la desviación típica, incluyendo el número total de valores válidos.

Para las comparaciones de subgrupos de pacientes, en el caso de variables cuantitativas, se utilizó la prueba paramétrica " $t$ de Student". Para las variables cualitativas se aplicó la prueba de la Chicuadrado. Los análisis estadísticos se realizaron con SAS versión 9.1.

\section{RESULTADOS}

La edad media (D.E.) de la muestra estudiada fue $59,2(12,9)$ años y el $73,6 \%$ eran mujeres. La edad media fue superior en los varones, 65,41 $(32,27)$ años, que en las mujeres, $57,94(20,38)$ años, $(p<0,001)$. Las características principales de la muestra estudiada se presentan en la Tabla I. La mayoría de las mujeres estudiadas $(71,9 \%)$ ya había tenido la menopausia, y tenían una media de 2,4 $(1,4)$ hijos, con $2,4(2,0)$ partos vaginales.

En la Tabla II se presentan las frecuencias de presentación de los diferentes trastornos crónicos estudiados para el total de la muestra y por separado para varones y mujeres con la significación estadística de la diferencia existente entre sexos. Entre los pacientes con diabetes fue más frecuente el tipo II 
$(86,1 \%)$ que el tipo I, no existiendo diferencias estadísticamente significativas $(p>0,05)$ para esta proporción entre varones y mujeres. Respecto a la cardiopatía isquémica, el infarto agudo de miocardio fue más frecuente en varones que en mujeres $(p<0,001)$, al igual que la angina $(p=0,001)$ y la revascularización coronaria $(p<0,001)$. El estreñimiento fue una patología que se presentó más frecuentemente en mujeres y la hipertensión, la diabetes, el accidente cerebro-vascular, la cardiopatía isquémica y las enfermedades respiratorias, en varones.

Según el cuestionario ICIQ-UI SF, 1.314 sujetos estudiados $(79,6 \%)$ presentaban síntomas de incontinencia urinaria asociados a $\mathrm{VH}$, siendo este porcentaje superior en mujeres $(83,9 \%)$ que en varones $(66,9)$. La puntuación media del cuestionario ICIQ-UI SF fue de $12,85(3,1)$ para el conjunto de la muestra, superior en mujeres, 13,03 $(3,1)$ que en varones, $12,23(3,04)(p<0,001)$.
La puntuación total en el ICIQ-UI SF fue superior en los pacientes que presentaban comorbilidad que en los que no la presentaban (Tabla III). Esto se observó también separando los sujetos por sexo.

En la Tabla IV se presentan las frecuencias de presentación de las patologías asociadas que se evaluaron en el presente estudio, para el total de la muestra y por separado para varones y mujeres con la significación estadística de la diferencia existente entre sexos. Las infecciones urinarias y de la piel del área genital y la depresión, fueron patologías que se presentaron más frecuentemente en mujeres y la disfunción sexual y la enfermedad neurológica, en varones, no existiendo diferencias según sexo en la frecuencia de presentación de caídas y trastornos del sueño. Atendiendo a la actividad sexual, el 60,5\% de los sujetos entrevistados manifestaron ser sexualmente activos, no existiendo diferencias entre varones $(60,9 \%)$ y mujeres $(60,4 \%)(p>0,05)$. Sin embargo,

TABLA I. CARACTERISTICAS PRINCIPALES DE LA MUESTRA ESTUDIADA.

\begin{tabular}{|c|c|c|}
\hline & $\mathbf{N}$ & $\%$ \\
\hline Ámbito de residencia & 1.651 & \\
\hline Rural $(<5.000$ habitantes $)$ & 184 & 11,1 \\
\hline Semiurbano (5.000-20.000 habitantes) & 356 & 21,6 \\
\hline Urbano ( $>=20.000$ habitantes) & 1.111 & 67,3 \\
\hline Nivel de estudios & 1.652 & \\
\hline Sin estudios & 235 & 14,2 \\
\hline Estudios primarios & 704 & 42,6 \\
\hline Estudios secundarios & 472 & 28,6 \\
\hline Estudios universitarios & 214 & 14,6 \\
\hline Situación laboral & 1.651 & \\
\hline Activo & 541 & 32,8 \\
\hline En paro & 39 & 2,4 \\
\hline Jubilado & 445 & 27,0 \\
\hline Incapacidad & 75 & 4,5 \\
\hline Ama de casa & 551 & 33,4 \\
\hline & Media & D.E. \\
\hline Peso (Kg) & 71,1 & 11,5 \\
\hline Talla $(\mathrm{cm})$ & 164,1 & 7,4 \\
\hline Perímetro de cintura $(\mathrm{cm})$ & 89,3 & 20,1 \\
\hline
\end{tabular}


TABLA II. FRECUENCIA DE PRESENTACIÓN DE TRASTORNOS CRÓNICOS CONCOMITANTES CON VEJIGA HIPERACTIVA.

\begin{tabular}{|l|c|c|c|c|}
\hline & $\begin{array}{c}\text { Total } \\
\mathbf{N}(\%)\end{array}$ & $\begin{array}{c}\text { Varones } \\
\mathbf{N}(\%)\end{array}$ & $\begin{array}{c}\text { Mujeres } \\
\mathbf{N}(\%)\end{array}$ & $\mathbf{p}$ \\
\hline Hipertensión & $699(42,3 \%)$ & $218(50,0 \%)$ & $481(39,5 \%)$ & $<0,001$ \\
\hline Diabetes & $368(22,2 \%)$ & $121(27,8 \%)$ & $247(20,3 \%)$ & 0,001 \\
\hline Accidente cerebro-vascular & $97(5,9 \%)$ & $48(11,0 \%)$ & $49(4,0 \%)$ & $<0,001$ \\
\hline Cardiopatía isquémica & $92(5,6 \%)$ & $50(11,5 \%)$ & $42(3,4 \%)$ & $<0,001$ \\
\hline Enfermedades respiratorias & $207(12,5 \%)$ & $95(21,8 \%)$ & $112(9,2 \%)$ & $<0,001$ \\
\hline Deterioro cognitivo & $38(2,3 \%)$ & $13(3,0 \%)$ & $25(2,1 \%)$ & 0,176 \\
\hline Cataratas o glaucoma & $136(8,2 \%)$ & $32(7,3 \%)$ & $104(8,5 \%)$ & 0,250 \\
\hline Disminución movilidad & $109(6,6 \%)$ & $27(6,2 \%)$ & $82(6,7 \%)$ & 0,396 \\
\hline Estreñimiento & $496(30,0 \%)$ & $59(13,5 \%)$ & $437(35,9 \%)$ & $<0,001$ \\
\hline
\end{tabular}

como puede verse en la Tabla IV, es muy superior el porcentaje de varones con disfunción sexual.

Las frecuencias de pacientes que asociaron las patologías estudiadas con los síntomas urinarios de $\mathrm{VH}$ se presentan en la Tabla $\mathrm{V}$ junto con el número medio con el que se presentaron durante el último año en el caso de las caídas y las infecciones urinarias de repetición y de la piel del área genital. La mayoría de los varones con disfunción sexual $(85,9 \%)$ tenían problemas de erección, siendo menos frecuentes la insatisfacción de la función eréctil $(30,0 \%)$, la ausencia de placer en las relaciones sexuales $(6,1 \%)$, o la ausencia de orgasmos $(6,6 \%)$. Sólo el $22,5 \%$ de los varones con disfunción sexual habían recibi- do tratamiento farmacológico para tratarla. Respecto a las mujeres lo más frecuente fue la falta 0 pérdida de deseo sexual $(82,9 \%)$, la falta o ausencia de placer $(32,9 \%)$ y el trastorno de la excitación sexual $(30,7 \%)$. El 54,3\% de los pacientes que tenían trastornos del sueño presentaban dificultad para conciliarlo, el $64,9 \%$ para mantenerlo varias horas seguidas, aunque tan sólo el 18,1\% experimentaban excesiva somnolencia durante el día.

\section{DISCUSIÓN}

En el presente estudio se han identificado patologías que los pacientes con $\mathrm{VH}$ presentan con frecuencia asociadas, entre ellas las infecciones en

TABLA III. PUNTUACIONES MEDIAS EN EL INTERNATIONAL CONSULTATION ON INCONTINENCE QUESTIONNAIRE URINARY INCONTINENCE SHORT FORM (ICIQ-UI SF).

\begin{tabular}{|l|c|c|c|}
\hline & Con comorbilidad & Sin comorbilidad & $\mathbf{p}$ \\
\hline Todos & $13,7(2,9)$ & $12,1(3,1)$ & $<0,001$ \\
\hline Varones & $12,9(2,9)$ & $11,7(2,9)$ & 0,001 \\
\hline Mujeres & $13,9(2,8)$ & $12,2(3,1)$ & $<0,001$ \\
\hline
\end{tabular}


TABLA IV. FRECUENCIA DE PRESENTACIÓN DE PATOLOGÍAS ASOCIADAS A LA VH.

\begin{tabular}{|l|c|c|c|c|}
\hline & $\begin{array}{c}\text { Total } \\
\mathbf{N}(\%)\end{array}$ & $\begin{array}{c}\text { Varones } \\
\mathbf{N}(\%)\end{array}$ & $\begin{array}{c}\text { Mujeres } \\
\mathbf{N}(\%)\end{array}$ & $\mathbf{p}$ \\
\hline Caídas & $167(10,1 \%)$ & $43(9,9 \%)$ & $124(10,2 \%)$ & 0,464 \\
\hline Infecciones urinarias & $553(33,5 \%)$ & $70(16,1 \%)$ & $483(39,7 \%)$ & $<0,001$ \\
\hline Infecciones en piel & $250(15,2 \%)$ & $35(8,1 \%)$ & $215(17,7 \%)$ & $<0,001$ \\
\hline Tratamiento antidepresivo & $252(15,3 \%)$ & $38(8,7 \%)$ & $214(17,6 \%)$ & $<0,001$ \\
\hline Disfunción sexual & $441(26,8 \%)$ & $213(49,0 \%)$ & $228(18,8 \%)$ & $<0,001$ \\
\hline Enfermedad neurológica & $148(8,9 \%)$ & $76(17,5 \%)$ & $72(5,9 \%)$ & $<0,001$ \\
\hline Incontinencia anal & $66(4,0 \%)$ & $18(4,1 \%)$ & $48(4,0 \%)$ & 0,480 \\
\hline Trastornos del sueño & $670(40,6 \%)$ & $180(41,4 \%)$ & $490(40,3 \%)$ & 0,372 \\
\hline
\end{tabular}

la piel del área genital, las infecciones del tracto urinario inferior y los trastornos del sueño, y que los pacientes más frecuentemente asocian a la presencia de síntomas de $\mathrm{VH}$. En los varones es más frecuente la disfunción sexual; y en las mujeres, las infecciones urinarias y de la piel del área genital y la depresión. El impacto en el paciente de estos trastornos, se evidencia por la mayor puntuación en el cuestionario ICIQ-UI SF de los pacientes que los padecen, respecto a los que no.

La mayoría de la muestra estudiada residía en ámbito urbano o semiurbano y tenían un nivel de estudios primarios o inferior. Las diferencias eviden- ciadas entre varones y mujeres en las frecuencias de presentación de las patologías concomitantes estudiadas, son las esperables en cualquier estudio epidemiológico: mayor frecuencia en varones en todas las patologías excepto en el estreñimiento que es más frecuente en mujeres y el deterioro cognitivo, las cataratas o glaucoma y la disminución de la movilidad que se presentaron en similar frecuencia relativa.

La diferente edad media de varones y mujeres con síntomas de $\mathrm{VH}$ incluidos en el presente estudio epidemiológico, confirma la diferente etiología de la patología en ambos sexos: en mujeres está más relacionada con problemas de suelo pélvico que em-

TABLA V. NÚMERO MEDIO DE PRESENTACIÓN DE CADA PATOLOGÍA ASOCIADA Y FRECUENCIA DE ASOCIACIÓN DECLARADA POR LOS PACIENTES, DE LAS DIFERENTES PATOLOGÍAS ESTUDIADAS CON LOS SINNTOMAS URINARIOS DE VEIIGA HIPERACTIVA CON SU INTERVALO DE CONFIANZA AL 95\%.

\begin{tabular}{|l|c|c|}
\hline Patología durante el último año & Número medio (D.E.) & Frecuencia de asociación (IC 95\%) \\
\hline Caídas & $1,8(1,1)$ & $26,9 \%(20,48-34,30)$ \\
\hline Infecciones urinarias de repetición & $3,4(2,2)$ & $66,4 \%(62,27-70,30)$ \\
\hline Infecciones en piel de área genital & $2,1(1,2)$ & $73,6 \%(67,60-78,86)$ \\
\hline Tratamiento antidepresivo & & $34,9 \%(30,2-39,5)$ \\
\hline Disfunción sexual & & $29,6 \%(25,41-34,15)$ \\
\hline Trastornos del sueño & & $57,8 \%(53,94-61,57)$ \\
\hline
\end{tabular}


piezan en edades medias de la vida y en varones se asocia con patología prostática fundamentalmente, cuya evolución se asocia al envejecimiento. Esta diferente edad media de varones y mujeres posiblemente también guarde relación con la diferente frecuencia de los trastornos crónicos estudiados, según el sexo. La proporción de mujeres con síntomas de IU añadidos a los de VH fue superior a la de varones: casi $80 \%$ de mujeres, frente al $67 \%$ de los varones. La mayor puntuación media en el cuestionario ICIQ-UI SF de mujeres, es indicadora de la mayor repercusión que los síntomas urinarios causan en este sexo.

Las patologías que se presentan con mayor frecuencia asociadas en los sujetos con $\mathrm{VH}$ del presente estudio son similares a los previamente identificados en otros estudios. Así ocurre con las infecciones del tracto urinario inferior (11), las infecciones de la piel (1 1), los trastornos del sueño (1 1), la depresión $(11,13,14$,$) y la disfunción sexual (11). Llama la$ atención sobre todo el elevado porcentaje de pacientes que atribuyen a los síntomas de $\mathrm{VH}$ la aparición de infecciones en piel de área genital y urinarias así como los trastornos del sueño, patologías todas que parecen presentarse con mayor frecuencia que en la población general. Por ejemplo los problemas crónicos de piel aparecen sólo en el $6 \%$ de sujetos de la población general entre 45 y 65 años (18) y la prevalencia de problemas de sueño en la población general, en el 22,8\% (19), lo que vendría a confirmar la asociación entre los síntomas de $\mathrm{VH}$ y estas patologías que consideran claras los pacientes al ser preguntados.

La presencia de estos trastornos se asocia a mayores puntuaciones (mayor importancia de los síntomas de IU) en el cuestionario ICIQ-UI SF, tanto en varones como en mujeres, por lo que parece relevante prestar atención a su detección en la historia clínica del paciente que consulta por síntomas de $\mathrm{VH}$ y/o IU.

\section{BIBLIOGRAFIA y LECTURAS RECOMENDADAS (*lectura de interés $y^{* *}$ lectura fundamental)}

1. Abrams P, Wein A J. The overactive bladder, a widespread but treatable condition. Stockholm Sparre Medical Group. 1998.

2. Elliot D, Lightner D, Blute M. Medical management of overactive bladder. Mayo Clin Proc 2001; 76: 353-355.

3. Sánchez R, Rupérez O, Delgado MA et al. Prevalencia de la incontinencia urinaria en la población mayor de 60 años atendida en atención primaria. Atención primaria 1999; 24: 241-248.

4. Abrams P, Cardozo L, Fall M et al. Thew standarisation of terminology of lower urinary tract func- tion: report from the Standarisation Sub-comitee of the International Continence Society. Neurology and Urodynamics, 2002; 21: 167-178.

5. Castro D, Espuña M, Prieto M, Badia X. Prevalencia de Vejiga Hiperactiva en España: Estudio poblacional. Arch Esp Urol, 2005; 58: 131-138.

6. Abrams P, Kelleher CJ, Kerr LA Rogers RG. Overactive bladder significantly affects quality of life. Am J Managed Care 2000; 6: 580-590.

7. Komaroff AL, Fagioli LR, Doolittle TH, Gandek B, Gleit MA, Guerriero RT, et al. Health status in patients with chronic fatigue syndrome and in general population disease comparison groups. Am J Med 1996; 101: 281-290.

8. Brocklehurst JC. Urinary incontinente in the community: analysis of a MORI poll. BMJ 1993; 306: 832-834.

9. Milsom I, Abrams P, Cardoza L et al. How widespread are the symptoms of overactive bladder and how are they managed? A Population-based prevalence study. BJU Int 2001; 87: 760-766.

10. Victor W. Nitti, MD. Clinical Impact of Overactive Bladder. Medreviews 2002; 4: 2-6.

11. Brown JS, McGhan WF, Chokroverty S. Comorbidities associated with overactive bladder. Am J Managed Care 2000; 6: 574-579.

12. Brown JS, Vittinghoff E, Wyman J, Stone KL, Nevitt MC, Ensrud KE, Grady D. Urinary incontinence: does it increase risk for fall and fracture? J Am Geriatr Soc. 2000; 48: 721-725.

13. Zorn BH, Montgomery H, Pieper K, Steers WD. Urinary Incontinence and depression. J Urol 1999; 162: 82-84.

14. Stach-Lempinen B, Hakala AL, Laippala $P$ et al. Severe depression determines quality of life in urinary incontinence women. Neurourol Urodyn 2003; 22: 563-568.

15. Darkow T, Fontes CL, Williamsom TE. Costs associated with the management of overactive bladder and related comorbidities. Pharmacotherapy. 2005; 25: 511-519.

16. Espuña M, Clota M, Rebollo P. Validación en España del cuestionario de autoevaluación del control de la vejiga hiperactiva en el primer nivel asistencial. Actas Urol Esp 2006; 30: 1017-1024.

17. Espuña M, Rebollo P, Puig M. Validación de la versión española del Internacional Consultation on Incontinente Questionnaire-Short Form. Un cuestionario para evaluar la incontinencia urinaria. Med Clin 2004; 122: 288-292.

18. Instituto Nacional de Estadística. Encuesta Nacional de Salud 2006. Disponible on-line: http:// www.ine.es $/$ jaxi $/$ menu.do?type $=$ pcaxis $\&$ path $=/$ t15/p419\&file=inebase $\& \mathrm{~L}=0$ Consulta el 10 de abril de 2008.

19. Vela-Bueno A, de Iceta M, Fernández C. Prevalencia de los trastornos del sueño en la ciudad de Madrid. Gaceta Sanitaria 1999; 13: 441-8. 